\title{
ARTICLE
}

\section{Handling of high resolution imaging data for usage in Monte Carlo calculations}

\author{
Uwe Reichelt ${ }^{*}$, Dorothea Sommer and Jürgen Henniger \\ Technische Universität Dresden, Institute for Nuclear and Particle Physics, Helmholtzstr. 10, Dresden, 01062, Germany
}

This paper discusses the handling of high resolution imaging data in Monte Carlo radiation transport algorithms. A solution for the necessary reduction of the size of the anatomical data is presented. It is discussed by means of memory reduction, influence to calculation performance and possible inaccuracies. Examples of calculated dose distributions are shown.

Keywords: Monte Carlo; imaging data; radiotherapy; nuclear medicine; internal dosimetry

\section{Introduction}

Imaging data, e.g. from Computer tomography (CT) and Positron Emission Tomography (PET), gains more and more importance at the dose estimation for patients in the oncologic treatment [1-2]. These data are used in nuclear and radiotherapeutical medicine for individual treatment planning and for dose verification, respectively. Furthermore, in radiobiological studies using animal models and for the post exposure dose estimation in radiation protection, these data can provide more reliable results than those achieved using conventional dose estimations like dose point kernels and S-values algorithms (e.g. see [3]).

Together with the progress of radiation detection systems and reconstruction methods, imaging data with enhanced spatial resolution and imaging space becomes available. It enables the identification of small anatomical structures and an exact description of the organ shapes. But also its size increases which pose a challenge for dose calculation and treatment planning systems. A reduction of the data size would be useful. But even taking into account that the real physical spatial resolution of the imaging device doesn't coincide with the discretisation size of the imaging data, algorithms simply averaging over several imaging pixels always result in a loss of information.

For the usage in radiation transport algorithms in radiobiology and radiation protection there are high resolution computational phantoms available, like the DigiMouse with $2 \times 10^{7}$ [4-5], the Visible Human with $2 \times 10^{7}$ [6] or the VIP-man with $3 \times 10^{9}$ tissue voxels [7]. Also here the development of generalised anatomic voxel phantoms provides more and more detailed data.

Hence, an algorithm keeping the spatial resolution in regions where it is necessary and reducing it elsewhere

*Corresponding author. Email: reichelt@asp.tu-dresden.de would be very helpful concerning the memory requirement in run time and the storage space as well as the time consuming handling of such huge data sets in the preparation process.

\section{Methods}

\subsection{Sources of geometric data}

For the simulation of radiation transport, a description of spatial inhomogeneities of the material is necessary to describe the differences of the interactions correctly. This can either be done using geometric figures [8] or real measuring and imaging data. In medical applications, Computer Tomography (CT) or magnetic resonance tomography (MRT) can be utilised to characterise the anatomy [9]. Modern systems are able to achieve high spatial resolutions and a very detailed image space, like the Hounsfield unit scale. This data is predestined for applications like individual treatment planning.

Because this data is only valid for an individual, generalised statements required e.g. in radiation protection are difficult to derive. For such tasks, so called voxel phantoms are in use. Each of them represents a particular group of individuals like children of a certain age or gender dependent adults. They have a much smaller imaging space, normally representing only the discrete organ definition of the different volume elements.

For both sources, the provided imaging space doesn't meet the requirements for the usage in radiation transport codes. Hence, an increase of information in general is necessary for instance to apply independent regions of interest, weight window techniques, average dose estimations, local radiation transport flags as well as the separation of material properties like different chemical compounds and inhomogeneous mass densities 
in one organs. So, besides the increase of experimental spatial resolution also advanced Monte-Carlo methods result in a large data volume that has to be treated.

\subsection{Algorithm for geometry handling}

In principle, there are two approaches for the treatment of the geometric description in radiation transport depending on the sampling algorithm used for the particle step length. The first is based on the passage through the surfaces of the current volume [10]. The second approach only retrieves the parameters connected with the actual particle location [11]. For the latter, an algorithm to determine the transport relevant properties depending on the particles space coordinates will be introduced. It is based on a three dimensional binary tree. So, each knot of this tree devides the corresponding volume into eight sub volumes of the same size. It splits the cuboid in each Cartesian direction. Each sub volume can then again be splitted into eight subs. By this regime, a chain of knots is generated.

For further explanation, input data as a three dimensional voxel matrix is assumed. As any averaging in the spatial domain shall be neglected, the binning size of each direction is the same as that of the original input data. But in principle, any geometric description can be used to build up the binary tree. Here, the binning is defined by the required spatial resolution of surfaces and the smallest dimension of included structures.

Because of the symmetry of the binary tree the algorithm provides the same number of voxels in each direction. To describe the whole original definition space without loosing information, the voxel number can only be increased. Hence, and because of the use of binary decisions, the number is defined by the the first potential of 2 that is larger than the highest number of discretisation intervalls of all dimensions. This procedure can result in a significantly enlarged definition space, but there is no data loss yet.
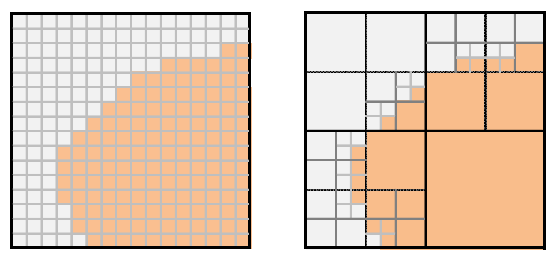

Figure 1. 2D scheme of data abstraction using the voxel tree.

The search algorithm runs step by step. Each time there is the decision if the coordinate in each direction is below or above the given cut value in the middle of the volume. So, there are eight sub volumes in the following level of the tree, and the algorithm choses one for the next step. Here, the same is done again until the final volume size is reached and the resulting transport parameters are determined. This algorithm leads to subvolumes like those shown in Figure 1 at the left side.

It is obvious that this chain can be stopped as soon as all following sub volumes return the same transport parameters. So it is possible to reduce the amout of data by $1: 8^{n}$ depending on the remaining steps $n$ until the end of the chain is reached (compare with the illustration at the right side of Figure 1).

The usage of binary decisions implies the usage of binary structures and operations for the search algorithm. Inside a search loop which runs over the different tree levels, the decision for the choice of the next sub volume is simply reproduced using bit operations combined with pointer arithmetics. This results in a fast implementation.

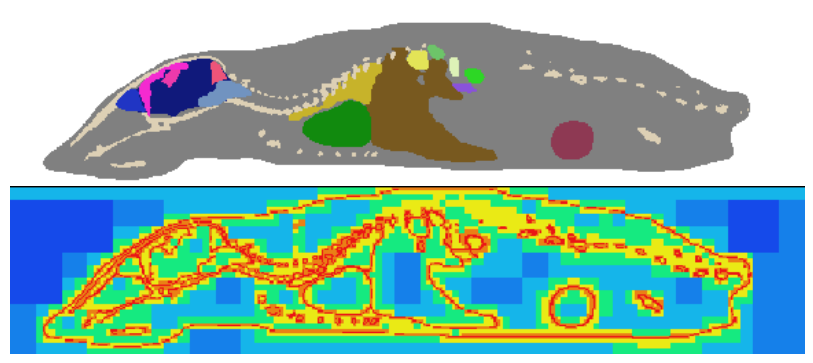

Figure 2. Anatomy of the Digimouse phantom [4-5] (top) and its subvolume representation (bottom).

\subsection{Criteria for further data reduction}

For quite homogenous voxel geometries like computational phantoms, this is a very efficient procedure compared to the application to noisy measuring data like CT-Data. For example, Figure 2 shows a phantom of the DigiMouse. The upper picture gives the cut view of the original data. Therein, several organs and tissues are defined. The one below presents the final depth of the search algorithm. Large areas without differences are concluded, seen by the blue squares in Figure 2. Other areas covering differences in anatomy require detailed reproduction. They show as small red squares defining the organ shapes as detailed as they are defined by the original input data.

At this point, also the increase of the space of the original data described in 2.2 should be discussed. Because all areas outside of the original geometry will return the same transport parameters, e.g. geometric escape, this algorithm concludes these sub volumes. It results in only a small rise of the data amount.

Using real noisy measuring data, this fusion is rarely possible. In addition, also some amount of data is necessary to organise the binary tree structure. Hence, without any conclusion, there would be a data increase.

Thus, an additional window technique was implemented. It allows merging and averaging of sub voxels as long as all of them stay inside the predefined window range beginning from the smallest size. Hence, the total number of sub volumes as well as the mean number of knots per search run is reduced. These windows are parameter specifically defined. So e.g. areas of different regions of interest cannot be merged as well as volumes of different organs and material composition respectively. But local mass density variations can be averaged as long as they fulfill the window criteria. Thereby, structures like organ shapes 
are kept as smooth and detailed as possible.

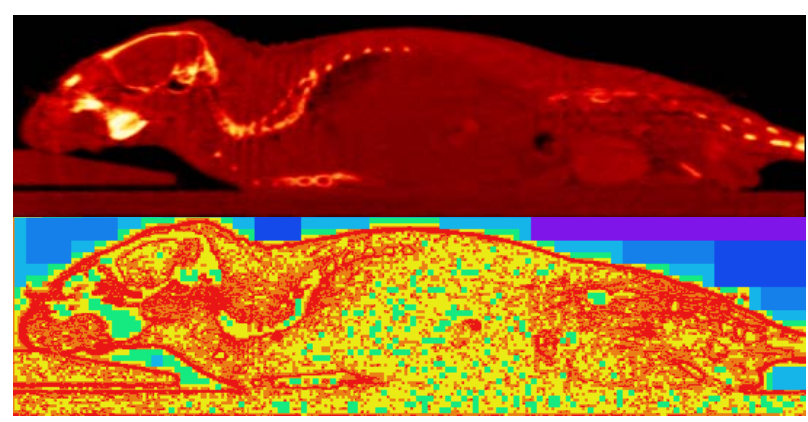

Figure 3. CT scan of mouse [7] (top) and its subvolume representation concerning a mass density window of $0.01 \mathrm{~g} / \mathrm{cm}^{3}$ (bottom).

An example shows Figure 3. Therein, the original CT data at the upper picture is compared to the sub volume clustering of the presented algorithm. At the borders between bone and soft tissue as well as at the body boundaries the voxel size decreases down to the original input pixel size. And also inside soft tissue there are many small regions that differ in their properties visible by the many small red clusters.

The efficiency of the merging of the sub volumes depends on the window size. For the example of Figure 3 a mass density window of $0.01 \mathrm{~g} / \mathrm{cm}^{3}$ is used. To familiarise with the influence of the window size, Table 1 shows the resulting file sizes. Of course, only small window sizes correspond to the uncertainties of the original input data and hence, are reasonable to apply. But even with a narrow window like $0.005 \mathrm{~g} / \mathrm{cm}^{3}$ the file size for this example can be reduced by $37 \%$ compared to the size of conventional matrix format, which is saved as a sequential binary matrix output. At very large window widths, the file size converges to a constant value of about $74 \mathrm{MB}$.

Table 1. Resulting file sizes depending on the mass density window range.

\begin{tabular}{ccc}
\hline $\begin{array}{c}\text { mass density } \\
\text { window } \\
\left(\mathrm{g} / \mathrm{cm}^{3}\right)\end{array}$ & $\begin{array}{c}\text { optimisation } \\
\text { depth } \\
n_{\text {od }}\end{array}$ & $\begin{array}{c}\text { resulting file } \\
\text { size } \\
(\mathrm{MB})\end{array}$ \\
\hline conv. Format & --- & 1123 \\
0.000 & 10 & $<32768^{*}$ \\
0.005 & 10 & 709 \\
0.010 & 10 & 699 \\
0.020 & 10 & 678 \\
0.040 & 10 & 584 \\
0.070 & 10 & 398 \\
0.100 & 10 & 255 \\
0.200 & 10 & 129 \\
0.500 & 10 & 74 \\
1.000 & 10 & 74 \\
10.000 & 10 & 74 \\
\hline theoretically calculated & &
\end{tabular}

For testing purposes geometries with limited optimisation depth were created. In the following, the depth $n_{\text {od }}$ means the number of levels that can be concluded starting from the most detailed representation. So, not more than $2^{n_{\text {od }}}$ subvolumes can be concluded in each tree branch. Table 2 shows the results for the example discussed before. It can be seen that the first two orders are extremely necessary to achieve the original data size and that the reduction converges quite fast around a depth of five. Larger cubic volumes are only found in the areas outside the original geometry, and even there, the reduction into higher levels doesn't significantly contribute to the total data amount.

Table 2. Resulting file sizes depending on the optimisation depth $n_{\text {od. }}$.

\begin{tabular}{ccc}
\hline $\begin{array}{c}\text { mass density } \\
\text { window } \\
\left(\mathrm{g} / \mathrm{cm}^{3}\right)\end{array}$ & $\begin{array}{c}\text { optimisation } \\
\text { depth } \\
n_{\text {od }}\end{array}$ & $\begin{array}{c}\text { resulting file } \\
\text { size } \\
(\mathrm{MB})\end{array}$ \\
\hline $\begin{array}{c}\text { conv. Format } \\
0.010\end{array}$ & -- & 1123 \\
0.010 & 1 & $32768^{*}$ \\
0.010 & 2 & 8793 \\
0.010 & 3 & 1708 \\
0.010 & 4 & 824 \\
0.010 & 5 & 714 \\
0.010 & 6 & 701 \\
0.010 & 7 & 699 \\
0.010 & 8 & 699 \\
0.010 & 9 & 699 \\
0.010 & 10 & 699 \\
\hline
\end{tabular}

*theoretically calculated

\subsection{Comparison with other radiation transport codes}

There are data reduction techniques for other Monte Carlo radiation transport codes, too. E.g. in MCNP, there is a method known as repeated structures or LATTICE card [12]. Therein, the geometric world or cell can be divided into periodical volumes. To each, one out of a set of transport properties is assigned. So there is a reduction of necessary data, too. But there is still the periodical division of the space down to the voxel size, that influences the simulation speed and limits together with the size of the codomain the saving in memory usage.

For the image-based whole body phantom, called VIP-Man, calculations up to 800 million voxel elements were reported [13]. But therein the codomain is restricted to a small discrete number of organs and tissues. That is not the case if medical imaging data is used, concerning the local variability of the mass density.

\section{Example for usage in radiobiology}

\subsection{Additional methods}

The described algorithm was implemented into a general data handling tool developed for data preparation for the Monte Carlo program code AMOS [14]. This radiation transport program offers the coupled electron photon transport as well as the neutron transport 
in the lower energy range and in different geometries. As physical source even complex decay chains [15] can be chosen which also enables a semi time dependent detector response. The program is written in $\mathrm{C}++$ standard and is designed to be run on conventional desktop computer systems.

The geometry tool is used for constructing geometries defined by geometric shapes as well as by voxel matrices. For the latter, it also supports the import of different standard file formats like DICOM and ECAT 7. This input data can be modified in sense of shifts and rotations to achieve correct geometric positioning as well as by segmentations to define regions of interest like organs.

A major advantage of the handling tool is that identical routines are used for visualisation as well as for the MC program. This fact is essential, concerning the verification of the input data and hence decreasing the risk of potential mistakes in the process of simulation preparation.

The following example is based on the CT (see Fig. 3, upper picture) and PET imaging data, which are derived from a standard nude mice model treated with ${ }^{86}$ Y-DTPA-Cetuximab [16]. These experiments were carried out in a joined cooperation project between physicians, biologists and physicists [17]. The applied radiopharmaceutical consists of the radionuclide ${ }^{86} \mathrm{Y}$ bound to diethylene triamine pentaacetic acid (DTPA). This complex is coupled to the antibody Cetuximab (C225), which binds to the Epidermal Growth Factor Receptor (EGFR). Different tumor cell lines show an enhanced expression of this receptor.

${ }^{86} \mathrm{Y}$ enables the imaging of the activity distribution via PET scan. This data provides the spatial source distribution inside the animal. With the help of the radiation transport simulation it is possible to study the influence of different radionuclides bound to the same radiopharmaceutical, assuming that the chemical and biochemical properties will not change significantly.

\subsection{Results}

In Figure 4 the results for the dose distribution are shown for ${ }^{177} \mathrm{Lu}$ and ${ }^{90} \mathrm{Y}$. The distributions are scaled to the maximum to achieve a better visualisation. But also for the practical usage this is reasonable, because the total amount of injected activity will be varied to achieve the necessary dose in the target tissue. It shall be mentioned that for both calculations the spatial resolution of the activity distribution and the detector matrix are the same. The difference can be seen clearly. The ${ }^{177} \mathrm{Lu}$ treatment gives a much more structured distribution than ${ }^{90} \mathrm{Y}$, because of the shorter maximal electron range. It is $1.7 \mathrm{~mm}$ in water compared to $10.8 \mathrm{~mm}$ of ${ }^{90} \mathrm{Y}$. For lutecium, even the squared structure originating from the assumed activity distribution is visible. It results from the relatively low resolution of the PET data.

Despite of the better localisation of the dose to the activity, ${ }^{177} \mathrm{Lu}$ emits photons as well. This results in a far distant dose deposition. The higher $\beta^{-}$range of ${ }^{90} \mathrm{Y}$ could be useful to achieve a more homogenous dose deposition inside massive tumors. In their centre, a significant leak of the activity concentration can occur, like in the case of reduced perfusion. This effect can be seen in Figure 4. At the lower right part the image plane cuts a massive tumor at the mouse leg. The ${ }^{90} \mathrm{Y}$ treatment results in a slightly inhomogenous dose deposition in the tumor with a minimum in the centre. The ${ }^{177} \mathrm{Lu}$ marked pharmaceutical gives a stronger underexposure there. This can necessitate the increase of the total applied activity and hence the dose to other organs.

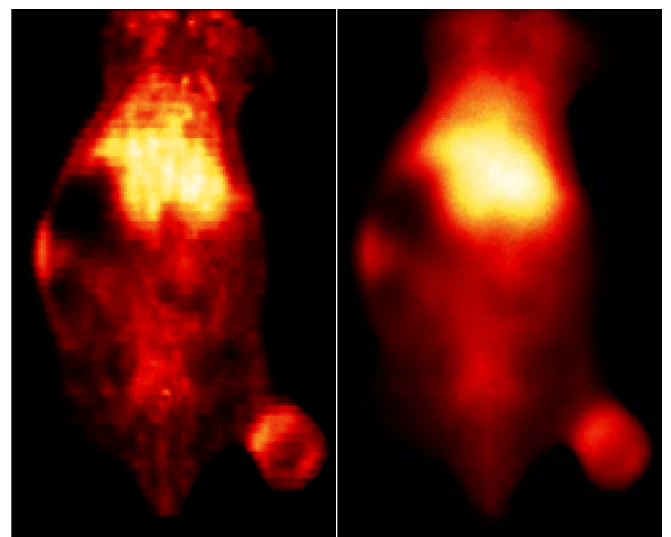

Figure 4. Dose distribution of injected radio pharmaceutical after defined time concerning different bound radionuclides, left: ${ }^{177} \mathrm{Lu}$ compound, right: ${ }^{90} \mathrm{Y}$ compound.

The usage of the voxel tree algorithm can result in a slightly longer runtime. In the discussed example, it was increased by $7 \%$ compared to the usage of a conventional matrix algorithm. This amount strongly depends on the simulated scenario. It decreases with the decreasing mean depth in the tree structure and therefore also with increasing number of conclusion of sub volumes. Furthermore, the independency of the spatial coordinates in the sequence of search runs influences the run time, taking into account internal caching algorithms of the CPU.

In general, there is no difference in the results between conventional simulation and applying the presented algorithm. Differences can only emerge if the density window is applied. To define the upper limit for its size an overall uncertainty analysis has to be done. A fast estimate for an appropriate size can be achieved by investigating the uncertainties in the provided imaging data and the derived mass density values. General values cannot be given, because of the dependence of the image quality on the chosen imaging protocol and imaging device. The straggling of the Hounsfield unit values can be determined with measurements using homogeneous phantoms.

\section{Conclusion}

In this paper an algorithm handling large geometric data sets for usage in radiation transport simulation is 
presented. It is shown that it enables significant memory savings in runtime and for storage. With it is possible to use high resolution imaging data as input data without the need of decreasing the spatial resolution. One advantage is to enable multiple calculations with different input data using the multi core technology of modern desktop systems. Furthermore in conventional desktop computing systems 32 bit operating systems are still very common. Thereby a single process is limited to 2GB of memory - a limit that cannot be shifted by simply upgrading the hardware.

\section{Acknowledgements}

The authors want to thank Dr. Bergmann from Hemholtz-Zentrum Dresden-Rossendorf for providing the authors with the imaging data for the shown example.

This work is part of a project funded by the Federal Ministry of Education and Research under sign 02NUK006E. The content is in responsibility of the authors.

Furthermore, the authors want to thank all partners of the project for the fruitful cooperation.

\section{References}

[1] B. Gehler, F. Paulsen, M. Öksüz, T. Hauser, S. Eschmann, R. Bares, Ch. Pfannenberg, $\mathrm{M}$. Bamberg, P. Bartenstein, C. Belka and U. Ganswindt, [68Ga]-DOTATOC-PET/CT for meningioma IMRT treatment planning, Radiat. Oncol. 4 (2009), pp. 56-64.

[2] H. Schöder, St. Larson and H. Yeung, PET/CT in Oncology: Integration into Clinical Management of Lymphoma, Melanoma, and Gastrointestinal Malignancies, J. Nucl. Med. 45 (2004), pp. 72S-81S.

[3] G. Sgouros, K. Kolbert, A. Sheikh, K. Pentlow, E. Mun, A. Barth, R. Robbins and St. Larson, Patient-specific dosimetry for 131I thyroid cancer therapy using 124I PET and 3-Dimensional-Internal Dosimetry (3D-ID) software, J. Nucl. Med. 45 (2004), pp. 1366-1372.

[4] B. Dogdas, D. Stout, A. Chatziioannou and R.M. Leahy, Digimouse: A 3D whole body mouse atlas from CT and cryosection data, Phys. Med. Bio. 52 (2007), pp. 577-587.

[5] D. Stout, P. Chow, R. Silverman, R. M. Leahy, X. Lewis, S. Gambhir and A. Chatziioannou, Creating a whole body digital mouse atlas with PET, CT and cryosection images, Mol. Imaging Biol. 4(4) (2002), p. 27.
[6] M. Zankl, U. Fill, N. Petoussi-Henss and D. Regulla, Organ dose conversion coefficents for external photon irradiation of male and female voxel models, Phys. Med. Biol. 47 (2002), pp. 2367-2385.

[7] The Visible Human Project, U.S. National Library of Medicine, www.nlm.nih.gov/research/visible (2012).

[8] M. Blaickner and P. Kindl, Diversification of existing reference phantoms in nuclear medicine: Calculation of specific absorbed fractions for 21 mathematical phantoms and validation through dose estimates resulting from the administration of (18)F-FDG, Cancer Biother. Radiopharm. 23(6) (2008), pp. 767-782.

[9] S. Marcatili, C. Pettinato, S. Daniels, G. Lewis, P. Edwards, S. Fanti and E. Spezi, Development and validation of RAYDOSE: a Geant4-based application for molecular radiotherapy, Phys. Med. Biol. 58 (2013), pp. 2491-2508.

[10]J. Durkee, MCNP geometry transformation and plotter equations, Prog. Nucl. Energy. 61 (2012), pp. 26-40.

[11]W. Colemane, Mathematical verification of a certain Monte Carlo sampling technique and applications of the technique to radiation transport problems, Nucl. Sci. Engin. 32 (1968), pp. 76-81.

[12]P. Ferrari and G. Gualdrini, An improved MCNP version of the NORMAN voxel phantom for dosimetry studies, Phys. Med. Biol. 50 (2005), pp. 4299-4316.

[13]B. Wang, X. G. Xu, J. T. Goorley and A. Bozkurt, Issues related to the use of MCNP code for an extremely large voxel model VIP-Man, Conf. Proc. Monte Carlo (2005), Chattanooga TN.

[14]U. Reichelt and J. Henniger, Application of advanced Monte Carlo methods in numerical dosimetry, Radiat. Protect Dosim. 119 (2006), pp. 479-482.

[15]D. Sommer, U. Reichelt, K. Helbig and J. Henniger, Nuclide decay simulation in AMOS, Proceedings of Jahrestagung Kerntechnik, Annual Meeting on Nuclear Technology (2011). [CD-ROM].

[16]R. Bergmann, CT- and PET imaging data, private communication, Hemholtzzentrum DresdenRossendorf (2011).

[17]J.-M. Heldt, M. Saki, J. Saker et al., In vitro and in vivo evaluation of yttrium-90 labelled cetuximab in combination with external X-ray irradiation radiooncology and nuclear medicine shake hands, Quarterly Journal of Nuclear Medicine and Molecular Imaging 56 (2012), p. 7. 